\title{
Development of new liquid scintillators for neutrino experiments of next generation
}

\section{Sultim Lubsandorzhiev ${ }^{\mathbf{1}}$}

Institute for Nuclear Research of the Russian Academy of Sciences

Moscow, pr-t 60-letiya Oktyabrya 7a, Russia

E-mail: sultim@inbox.ru

\section{Andrey Sidorenkov}

Institute for Nuclear Research of the Russian Academy of Sciences Moscow, pr-t 60-letiya Oktyabrya 7a, Russia

E-mail: andreassx7@gmail.com

\section{Bayarto Lubsandorzhiev}

Institute for Nuclear Research of the Russian Academy of Sciences Moscow, pr-t 60-letiya Oktyabrya 7a, Russia

E-mail:lubsand@rambler.ru

\section{Sergey Ponomarenko}

Institute for Nuclear Research of the Russian Academy of Sciences Moscow, pr-t 60-letiya Oktyabrya 7a, Russia

E-mail: andreassx7@gmail.com

\section{Nikolay Surin}

Institute for Nuclear Research of the Russian Academy of Sciences Moscow, pr-t 60-letiya Oktyabrya 7a, Russia

E-mail: andreassx7@gmail.com

\section{Oleg Borshev}

Institute for Nuclear Research of the Russian Academy of Sciences Moscow, pr-t 60-letiya Oktyabrya 7a, Russia

E-mail: andreassx7@gmail.com

\section{Nikita Ushakov}

Institute for Nuclear Research of the Russian Academy of Sciences Moscow, pr-t 60-letiya Oktyabrya 7a, Russia

E-mail: nikita.ushakoff@gmail.com

\section{Nima Lubsandorzhiev}

Scobeltsyn Institute of Nuclear Physics of Lomonosov Moscow State University

Moscow, Leninskie gory 1, Russia

E-mail: nilubs@rambler.ru 


\title{
Dmitriy Voronin
}

Institute for Nuclear Research of the Russian Academy of Sciences Moscow, pr-t 60-letiya Oktyabrya 7a, Russia

E-mail:dmitriy.voronin@gmail.com

\section{Arslan Lukanov}

Institute for Nuclear Research of the Russian Academy of Sciences

Moscow, pr-t 60-letiya Oktyabrya 7a, Russia

E-mail: arslanlukanov@yandex.ru

\section{Almaz Fazliakhmetov}

Institute for Nuclear Research of the Russian Academy of Sciences Moscow, pr-t60-letiya Oktyabrya 7a, Russia

E-mail: andreassx7@gmail.com

\begin{abstract}
We present results of development of new liquid scintillators for neutrino experiments of next generation. Liquid scintillators are working horses for neutrino experiments starting from the very beginning of neutrino physics. Indeed experimental discovery of neutrino was done by using liquid scintillator. Liquid scintillators have been intensely used in neutrino physics experiments like Borexino, KamLAND, SNO, Double Chooz, Daya Bay, RENO etc. Presently a number of new experiments in the field are being constructed or under development like JUNO, $\mathrm{SNO}+$, RENO-50 etc.
\end{abstract}




\section{Introduction}

Liquid scintillators have been always playing a special role in experiments in astroparticle physics, in neutrino physics in particular. Indeed, even experimental discovery of neutrinos was done by F. Reines and C. Cowan using liquid scintillator detector [1]. In fact it was first use of relatively large amount of liquid scintillators and photomultipliers. Since that time liquid scintillators became a "workhorse" of physics experiments. Nowadays we are witnessing a real renaissance of liquid scintillators. As illustrations of the claim it is enough to mention three reactor neutrino experiments (Daya Bay, Double Chooz and RENO) based on liquid scintillator detectors which discovered non-zero value of the third mixing angle $\theta_{13}$ of neutrino oscillation mixing matrix or three more complex liquid scintillator detector experiments (Borexino, KamLAND and SNO+) making a crucial contributions to neutrino oscillation, solar neutrino physics, geoneutrinos, neutrinoless double beta-decay etc. Presently one of the most ambitious experiments in neutrino physics JUNO [2] is under construction in China. The amount of liquid scintillators in astroparticle experiments varies from dozens of tons in reactor experiments to 30 $\mathrm{kt}$ scale in case of JUNO experiment.

Physics parameters of liquid scintillators are crucial issue for experiments. The parameters like emission spectrum, light yield, emission kinetics etc in turn depends strongly on organic solvent (base or bulk material), scintillation dopants or fluors used in scintillators [3]. The most commonly used approach in liquid scintillators is to use two fluors. The first one absorbs excitation energy deposited in organic solvent by ionizing particle. The second one is wavelength shifting fluor to match well scintillation emission spectrum with scintillator's transparency. The base of scintillator, i.e. organic solvent, should provide high efficiency of transfer of excitation energy to the first scintillation fluor. It means that the solvent should have sufficiently high content of aromatics (benzene-rings) with delocalized clouds of $\pi$-electrons providing necessary high efficiency of energy transfer. At the same time the solvent has to be transparent, to have high flashing point for safety reasons. Finally, last but not least it should be cheap. The last points are very important for developing any large-scale experiments in astroparticle physics.

We developed new scintillation fluors to be used as a single fluor in LS. Such an approach allows increasing efficiency of fluors because there are no losses from excitation transfer from the first fluor to the second one. It is possible also to tune emission spectrum of new fluors to match it to LS transparency and photodetectors sensitivity. In this paper we present results of extensive studies of properties of developed fluors: emission spectrum, light yield, emission kinetics, solubility in different organic solvents, stability etc. We did some comparative studies of LAB based liquid scintillators using PPO+bis-MSB and new fluors. For some new fluors we observed two times higher light yield in $\mathrm{LAB}$ based liquid scintillators relative to traditional PPO+bis-MSB system

\subsection{Liquid scintillators (standard recepy)}

The most actively used organic solvent is linear alkilbenzene widely known as LAB. It is aromatic solvent with chemical formula $\mathrm{C}_{6} \mathrm{H}_{5} \mathrm{C}_{n} \mathrm{H}_{2 n+1}$ where $\mathrm{n}=10-16$. Its chemical structure shown in Fig.1. LAB has high flash point of more than $140^{\circ} \mathrm{C}$, it is relatively cheap (at the level 
of 2-3 USD per liter) and technologically well advanced - it is produced in huge amount all around the world mainly as a detergent. Further it is rather transparent. So LAB satisfies practically to all requirements for solvent for liquid scintillator applications.

\section{$-\mathrm{CH}_{2}-\mathrm{C}_{\mathrm{n}} \mathrm{H}_{2 \mathrm{n}-1}, \mathrm{n}=8-13$}

Fig.1. Chemical structure of linear alkyl benzene.

There are some problems with LAB's compatibility with other materials but they are overcome by careful choosing materials.

The most popular fluors for liquid scintillators are PPO as the first fluor together with small amount of bis-MSB (or POPOP) as the second wavelength shifting dopant. The concentration of the first fluor in solvent is at the level of $0.2-0.3 \%$ by weight $(2-3 \mathrm{~g} / \mathrm{l})$, whereas the amount of the second even tinier $-0.002-0.003 \%$ by weight $(\sim 20-30 \mathrm{mg} / \mathrm{l})$. In fig. 2 . emission spectra of PPO and bis-MSB are shown.

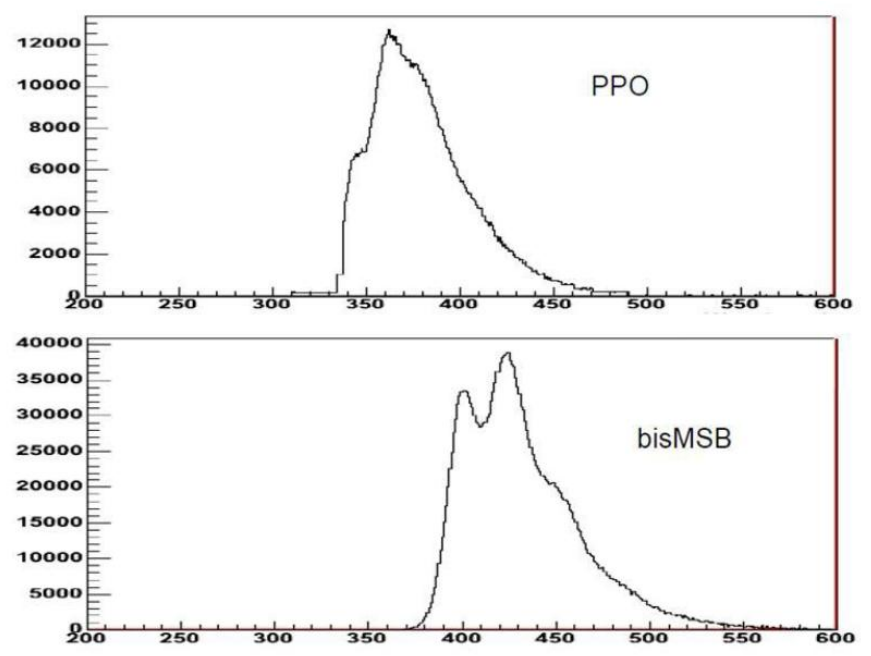

Fig.2. Emission spectra of PPO (up) and bis-MSB (bottom).

Emission spectrum of PPO lies in the near UV region with maximum at $\sim 360 \mathrm{~nm}$. PPO emission absorbed by bis-MSB which reemits light in violet region with maximum at 420-430 $\mathrm{nm}$. Of course in such two steps process energy losses are inevitable. There are other losses due to small Stocks shift in both PPO and bis-MSB, for the latter it is demonstrated in fig.3. So self absorption is rather high. Moreover at the maximum of emission of bis-MSB light absorption of raw LAB is still substantial, see fig.4 [4]. 


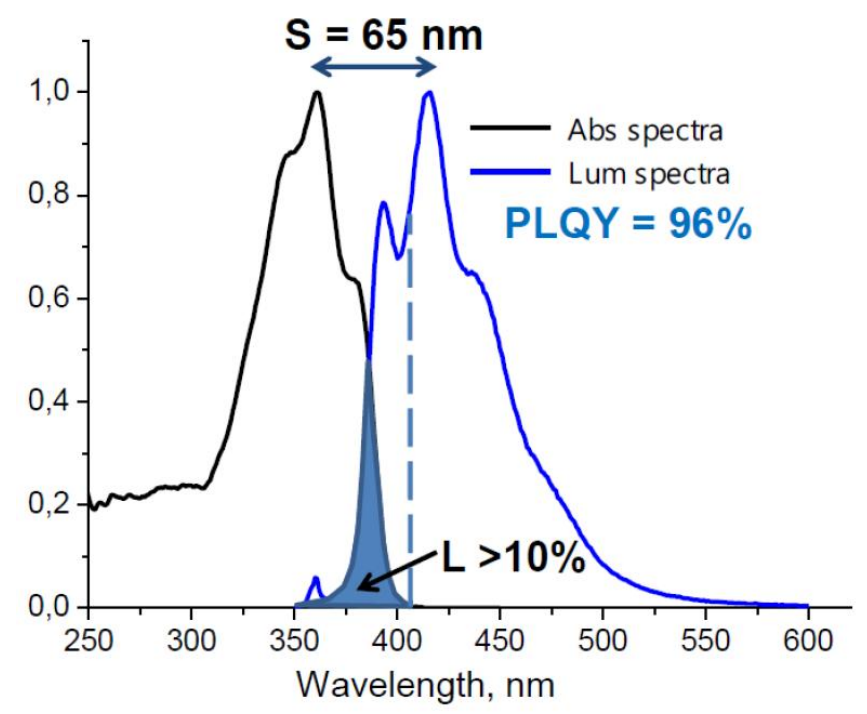

Fig.3. Absorption and emission spectra of bis-MSB.

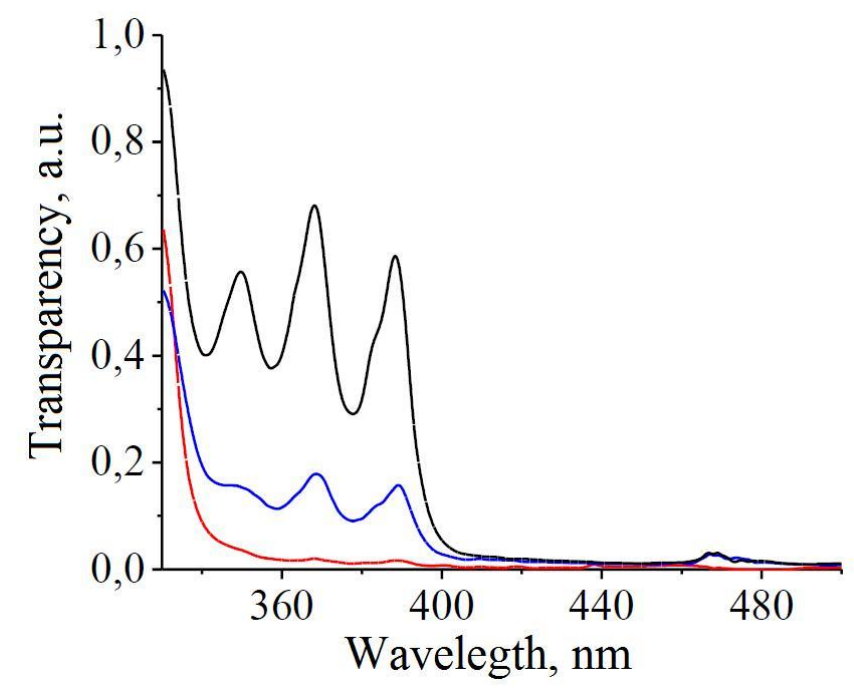

Fig.4. Transperancy of LAB before (black line) and after (red line) purification, from [4]

It results in need to construct sophisticated purification systems in order to get liquid scintillators with high enough transparency which is an absolute necessity for next generation large scale experiments in astroparticle physics. In future it may put problems for experiments.

How to overcome such problems? To do it we developed a number of new scintillation fluors which differs from "two-steps" approach mentioned above briefly. In our case we have one fluor instead of two. So, one fluor serves as fluor (e.g. PPO) and wavelength shifter (e.g. bis-MSB) simultaneously. A scheme roughly describing the principle is shown in fig.5. Here silicon-organic compounds are used. Donor molecules with high fraction of $\pi$-electrons are attached to the acceptor molecule from both sides by silicon bonding. 


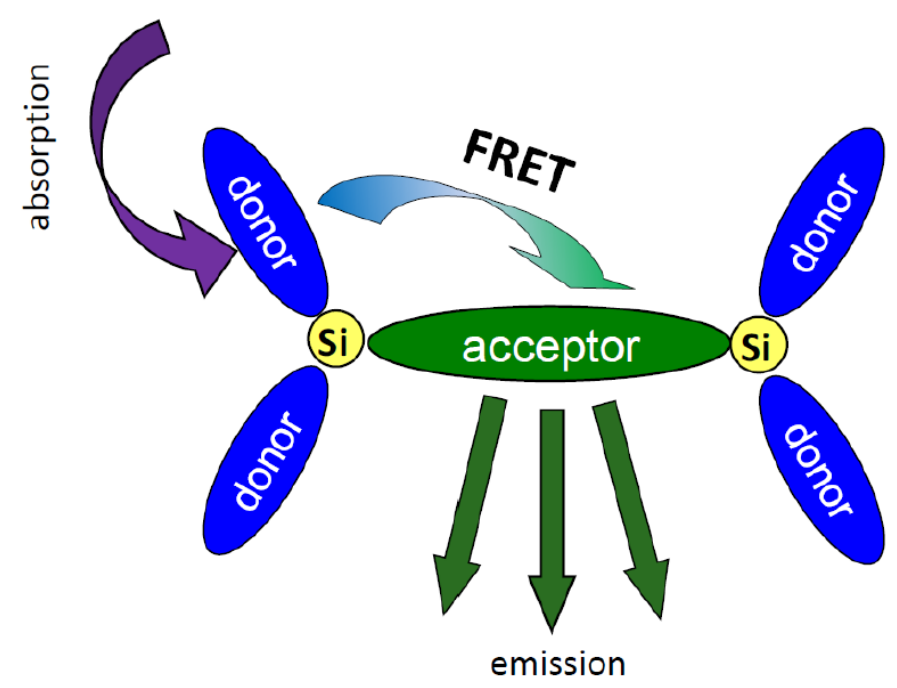

Fig. 5. A scheme of new scintillation fluors.

Excitation energy is absorbed by donor molecules and transferred via silicon molecules to acceptor molecules which in their turn emit light. Such an approach provides high efficiency of ionization energy transfer to scintillation light with high value of Stocks shift of up to $200 \mathrm{~nm}$, compare it with standard approach, see fig.3.

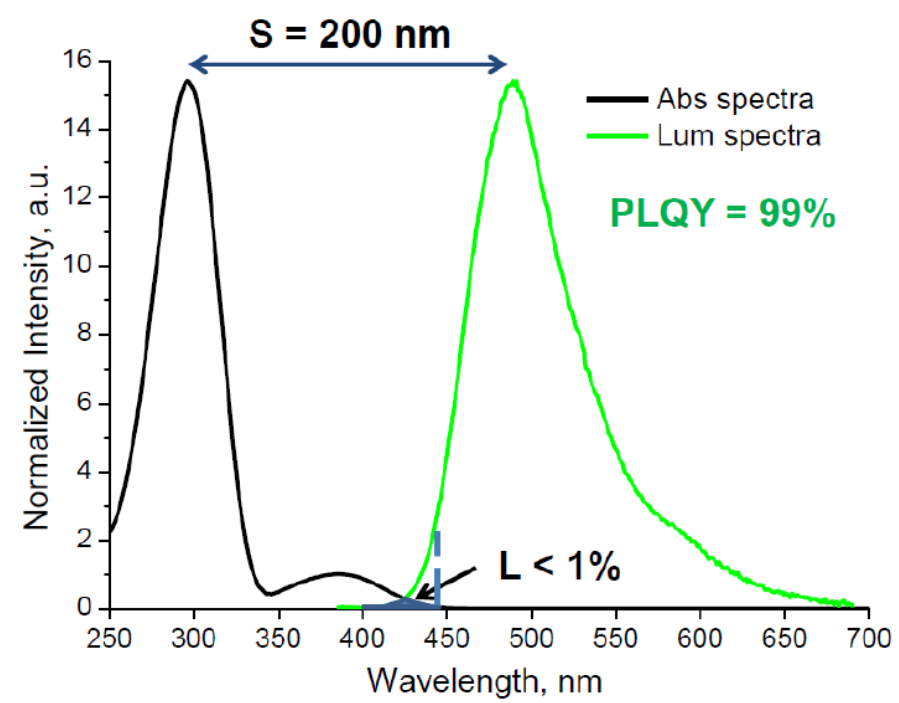

Fig. 6. Typical absorption and emission spectra of newly developed scintillation fluors.

A variety of chemical structures for new fluors are available, see fig.7. As one can see all structures are organo-silicon structures. 


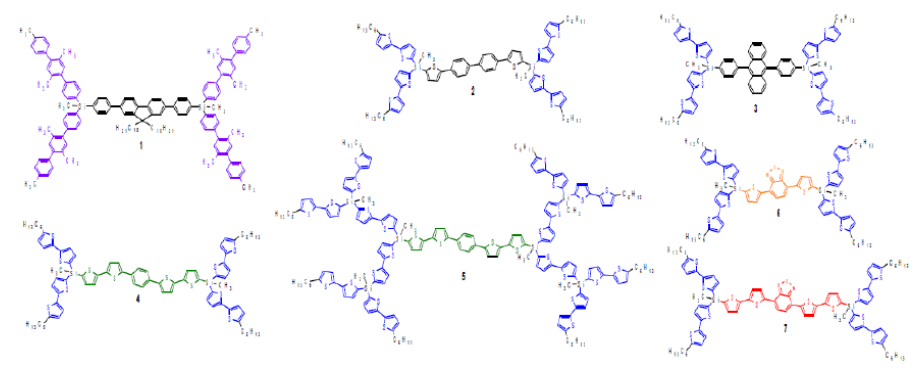

Fig. 7. Possible chemical structures of new fluors.

There is a mismatch of emission spectrum of new fluors and sensitivity curves of contemporary bialkali photocathodes usually used in photomultipliers but it is possible to use multialkali photocathodes with their sensitivity maximum exactly at the maximum of emission spectrum of the fluors, at $\sim 500 \mathrm{~nm}$. So, there are rooms for tuning photomultipliers sensitivity to match emission spectra of new fluors.

Moreover with new fluors new possibilities appear namely possibilities to use raw solvents because emission spectra with maximum at $\sim 490-500 \mathrm{~nm}$ are in the region where transparency of even raw non-purified solvents is high.

We developed liquid scintillators based on LAB as solvent and equipped with one of new fluors, called Skm219. Extensive studies of scintillation parameters of samples of new liquid scintillator are carried out. The samples has rather high light yield.

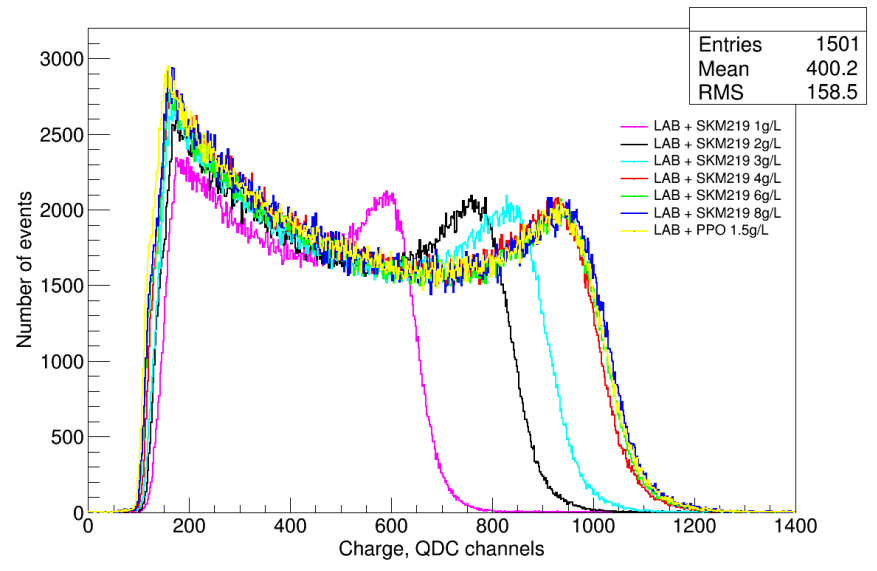

Fig. 8. Results of light yield measurements of liquid scintillators with new fluor under irradiation by $662 \mathrm{keV}$ gamma-quanta from ${ }^{137} \mathrm{Cs}$ radioactive source.

The spectra were measured by photomultiplier with high quantum efficiency bialkali photocathode. Taking into account sensitivity spectral curve the light yield of liquid scintillators with new fluors have almost two times higher light yield.

The liquid scintillators with new flours have slower light emission kinetics in comparison with "standard" scintillators with PPO+bis-MSB but not dramatically, as shown in fig.9. 


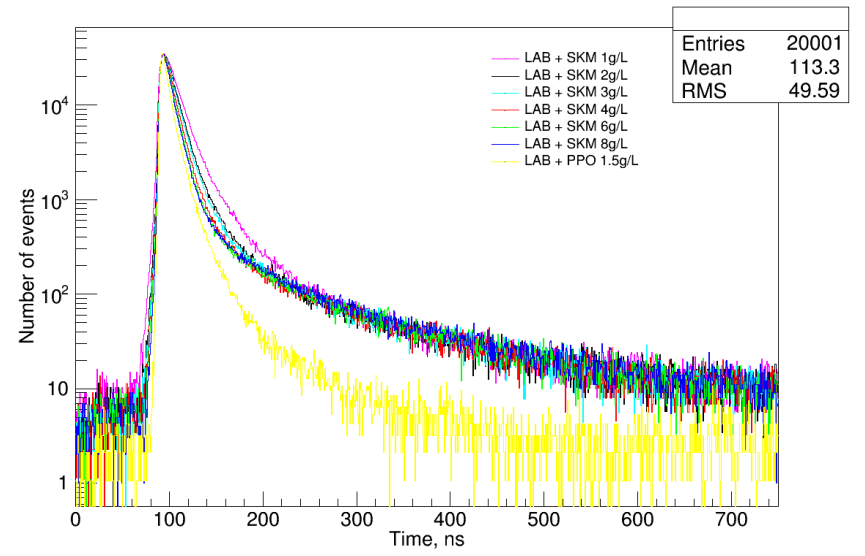

Fig. 9. Light emission kinetics of liquid scintillators with new fluors.

In Fig. 10 one can see a picture of samples of new LAB based liquid scintillators with new fluors.

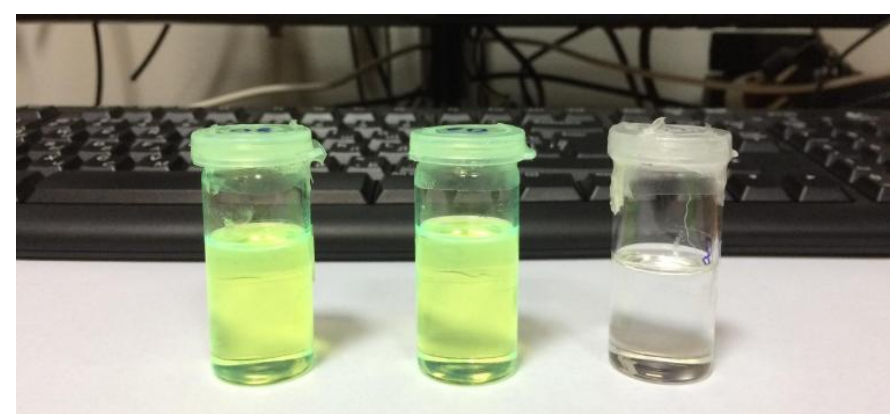

Fig. 10. Two samples of liquid scintillators with new fluors (in the right and center) and "standard" scintillator with PPO (in the left).

\section{Conclusion}

We developed new single fluors for liquid scintillation detectors for large-scale experiments of the next generation in astroparticle physics experiments. We also developed samples of liquid scintillators equipped with newly developed fluors. Their extensive studies show very promising results.

\section{Acknowledgements.}

The work was supported by the Russian Science Foundation grant no. 17-12-01331.

\section{References}

[1] C.L.Cowan, F.Reines et al. Detection of the Free Neutrino: a Confirmation // Science. 1956. Vol.124. P.3212.

[2] T. Adam et al. (JUNO Collaboration). JUNO conceptual design report // arxiv:1508.07166.

[3] J.B. Birks, Scintillation counters, London. 1953.

[4] I.B. Nemchenok. Habilitation Thesis. JINR, Dubna. 2019. 